\title{
Silver-negative NORs in Pamphagus ortolaniae (Orthoptera: Pamphagidae)
}

\author{
Roberto VITTURI ${ }^{1}$, Antonella LANNINO $^{1}$, CAterina MANSUETO $^{1}$, VAlentina MANSUETO $^{1}$ and Mariastella \\ COLOMBA $^{2 *}$
}

\author{
${ }^{1}$ Dipartimento di Biologia Animale, Università di Palermo, Via Archirafi 18, 90123 Palermo, Italy \\ ${ }^{2}$ Istituto di Ecologia e Biologia Ambientale, Università di Urbino, Via Maggetti 22, 60129 Urbino (PU), Italy; \\ e-mail: m.colomba@uniurb.it
}

Key words. Ag-NOR, FISH, chromosome, rDNA, telomere, Pamphagus ortolaniae, Orthoptera, Pamphagidae

\begin{abstract}
The present paper reports some cytogenetic peculiarities observed in the Ag-NORs of Pamphagus ortolaniae chromosomes, the unusual behaviour of ribosomal sites after silver staining and the intense Ag-positive reaction of centromeric regions at spermatogonial metaphase and spermatocyte metaphase I and II. Moreover, a conclusive identification and localization of all the ribosomal clusters is provided by using heterologous rDNA FISH on spermatocyte chromosomes. 18S-28S rDNA mapped on a single chromosome pair and resulted multiclustered along the chromosomal body in three distinct serial regions, $r_{1}, r_{2}$ and $r_{3}$. Surprisingly, these areas were scarcely $\left(r_{1}\right)$ or never $\left(r_{2}\right.$ and $\left.r_{3}\right)$ detectable by silver impregnation. As in other Orthoptera and many groups of arthropods, FISH with the pentamer $(\text { TTAGG })_{n}$ as the probe labelled the telomeres of all chromosomes.
\end{abstract}

\section{INTRODUCTION}

Nucleolus organizer regions (NORs) are characterized by the presence of clusters of tandemly repeated rDNA units, which when transcriptionally active are associated with nonhistone proteins (Miller et al., 1976; Jordan, 1987). The methods commonly used to detect NORs in animals and plants employ silver nitrate (Ag-NOR) or chromomycin $\mathrm{A}_{3}\left(\mathrm{CMA}_{3}\right)$ staining. Silver staining reveals transcriptionally active NORs by binding to nucleolar argentophilic acidic proteins; $\mathrm{CMA}_{3}$ generally shows both active and inactive NORs as a consequence of the high GC content of the rDNA.

However, mostly in invertebrates, both methods frequently do not work when applied to species belonging to taxonomically unrelated taxa. For example, silver nitrate does not stain NORs in the amphipod Jassa marmorata (Crustacea) (Libertini et al., 2000) and slug Milax nigricans (Mollusca) (Vitturi et al., 2004). On the other hand, silver and/or $\mathrm{CMA}_{3}$ heavily stain not only the NORs but also the heterochromatic blocks in the Algerian hedgehog Erinaceus (Aethechinus) algirus (Sánchez et al., 1995), ant Tapinoma nigerrimum (Lorite et al., 1997) and some scarab beetle species (Colomba et al., 2004 and references therein).

The genus Pamphagus (Orthoptera: Pamphagidae) includes four Mediterranean species, which occur in Sardinia (P. sardeus Herrich-Schaffer, 1840), Sicily (P. marmoratus Burmeister, 1838), Pelagie islands (P. ortolaniae Cusimano \& Massa, 1975) and North-Western Africa $(P$. tunetanus Vosseler, 1902). These species are morphologically similar but reproductively isolated and have been studied cytologically with respect to chromosome numbers ( $\mathrm{n}$ and $2 \mathrm{n}$ ) and C-banding patterns (Vitturi et al.,
1993 and references therein). In P. ortolaniae, a preliminary analysis using silver impregnation (Mansueto \& Vitturi, 1989) revealed that all spermatogonial chromosomes showed Ag-aggregates in their centromeric regions and, as a consequence, it was not possible to conclusively map the major ribosomal sites (18S-28S rDNA) and estimate their activity.

Fluorescence in situ hybridization (FISH) has considerable advantages over traditional systems for the specific and reproducible mapping of genes and DNA sequences. To date, it has been widely used for the localization of specific DNA sequences within the chromosomes (Swiger \& Tucker, 1996; Nath \& Johnson, 2000) and for examining major chromosome rearrangements (i.e. inversions and Robertsonian fusions) in a variety of organisms (e.g.. Slijepcevic, 1998; Nomoto et al., 2001). FISH along with the use of ribosomal sequences as probes has been successfully employed to provide unambiguous chromosomal assignments of major ribosomal sites in many species of grasshoppers in a broad range of taxa within the order (the huge number of papers published on this subject cannot be cited; see Souza et al., 1998, López-León et al., 1999; Cabrero et al., 2003; Keller et al., 2006; Souza $\&$ Melo, 2007 and references quoted therein).

In the present paper ribosomal clusters and telomeric sequences are mapped using FISH. Moreover, unusual outcomes such as the intense Ag-staining of centromeric regions during spermatogonial metaphase and metaphase I, along with the peculiar response of rDNA regions to silver staining, are discussed.

\section{MATERIAL AND METHODS}

Fifteen sexually mature males of the grasshopper Pamphagus ortolaniae (Orthoptera: Pamphagidae), identified using Cusi-

\footnotetext{
* Corresponding author.
} 

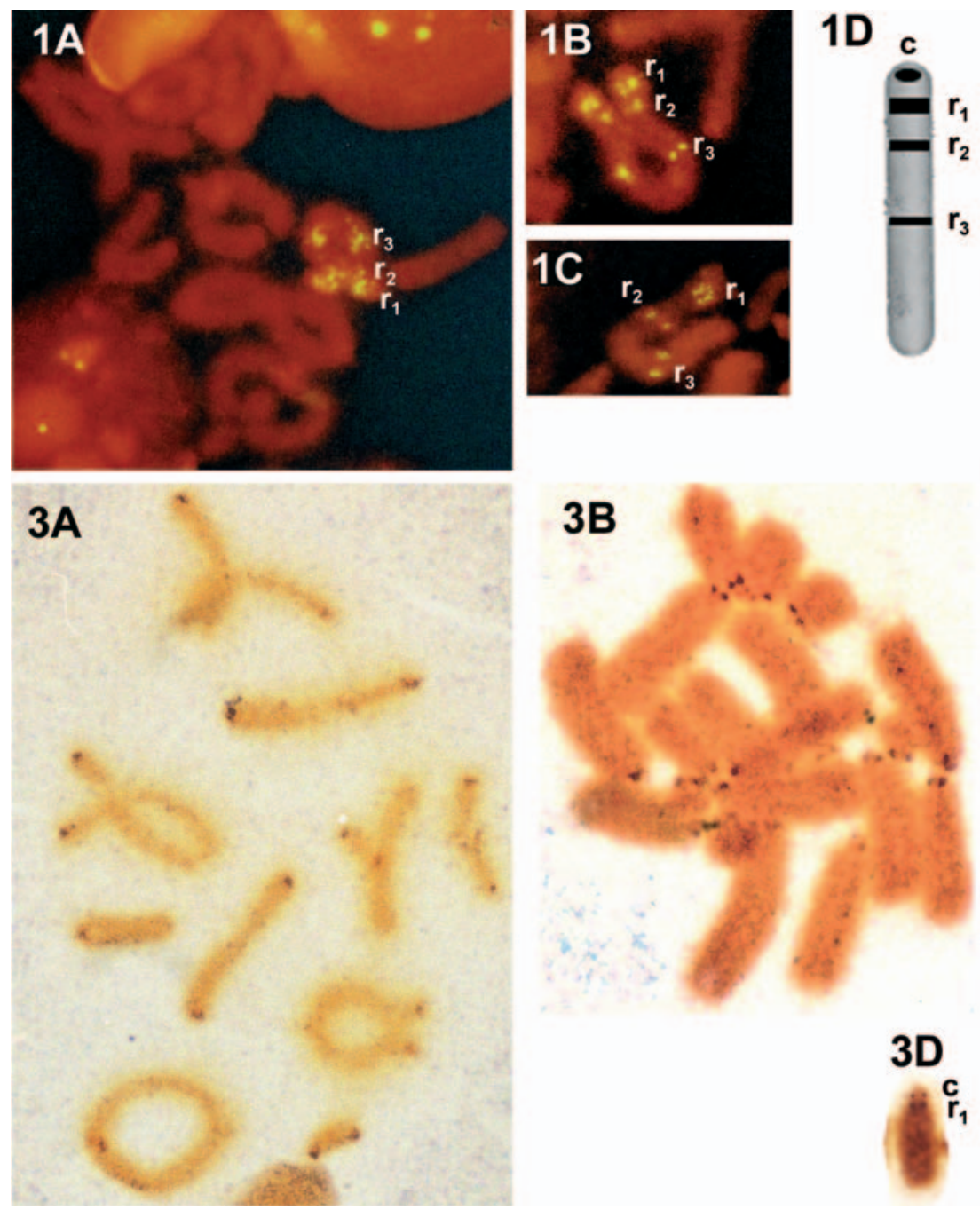
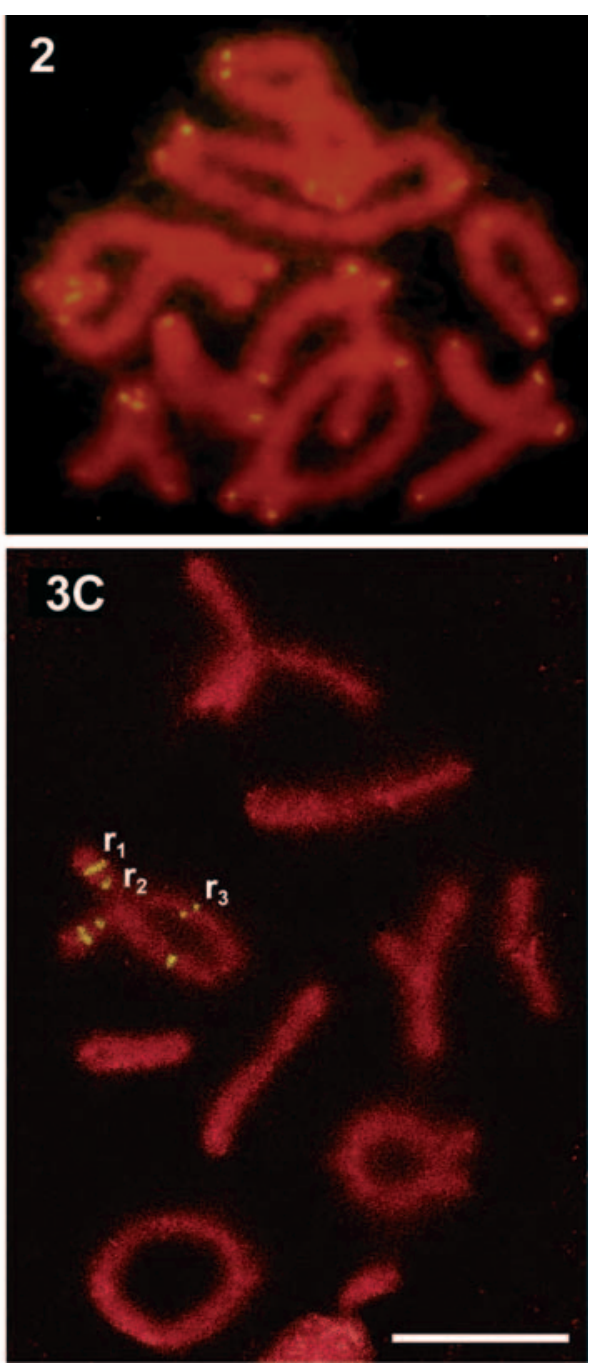

Figs 1-3: Localization of rDNA/NOR sites (1 and 3) and telomeric repeats (2) on chromosomes of Pamphagus ortolaniae. 1A Diakinetic bivalents after fluorescence in situ hybridization with $18 \mathrm{~S}$ rDNA from Paracentrotus lividus (sea urchin). Hybridized areas (yellow) consist of grains more or less closely joined, clustered in three regions $\left(\mathrm{r}_{1}, \mathrm{r}_{2}\right.$ and $\left.\mathrm{r}_{3}\right)$. 1B - The rDNA bearing bivalent from a second diakinetic chromosome plate hybridized with $18 \mathrm{~S}-5.8 \mathrm{~S}-28 \mathrm{~S}$ ribosomal probe from Drosophila melanogaster. The three serial regions are very evident. 1C - The rDNA bearing bivalent from a third chromosome plate at diplotene hybridized with the ribosomal probe from $P$. lividus. Hybridization patterns were always similar when two ribosomal probes prepared from taxonomically distant organisms, such as an echinoderm and an insect, were used. 1D - Schematic drawing of the NOR-bearing mitotic chromosome showing the distribution of rDNA signals $\left(r_{1}, r_{2}\right.$ and $\left.r_{3}\right)$; $c$ indicates the centromere position. 2 - Diakinetic bivalents after fluorescence in situ hybridization with the (TTAGG) ${ }_{n}$ telomeric probe. As in other arthropods, including other insects as well as P. ortolaniae, all chromosome ends are composed of pentamer repeats (yellow dots). 3 - Diakinetic bivalents (A) and spermatogonial metaphase chromosomes (B) after silver impregnation. Centromeric regions consistently show positive reaction to silver (dark dots) whereas rDNA clusters do not. Only in a few plates was the NOR-bearing chromosome pair marked by silver nitrate in a similar way to the ribosomal cluster $r_{1}$ and the centromeric region (c). (D) shows one of the homologous chromosomes on which the Ag-positive areas are clearly evident. (C) shows the same chromosome plate as in 3A after rDNA FISH. The sequential treatment with silver staining (A) and rDNA FISH (C) shows the dissimilarity of the two patterns. Scale bar $=10 \mu \mathrm{m}$ (except in 1D).

mano \& Massa (1977), were caught during July in 2005 and 2006 in fields on Lampedusa (Pelagie islands, Agrigento Province, South-Western Sicily).

Chromosomes were obtained by the air-drying method (Vitturi, 1992) from testicular lobes after in vivo colchicine treatment $(0.5 \mathrm{mg} / \mathrm{ml}$ for $1 \mathrm{~h})$, observed under a Leica microscope, photographed using a Kodak Ektacolor 800 ASA film and classified according to Levan et al. (1964). Characterization of nucleolus organizer regions (NORs) was done using the rapid colloidal silver method of Howell \& Black (1980).

FISH was performed on fixed spermatocyte chromosomes using four different probes: (1) a sea urchin (Paracentrotus lividus) 18S rDNA probe (Cantone et al., 1993) provided by R. Barbieri, University of Palermo; (2) a Drosophila melanogaster 18S-5.8S-28S rDNA probe (Roiha et al., 1981) kindly provided by J. Méndez and A. Insua, Universidade da Coruña, Spain; (3) the telomeric hexamer (TTAGGG) $)_{n}$ and (4) the insect-type pentamer repeat (TTAGG) $)_{n}$. The telomeric probes were obtained by PCR in the absence of a template (Ijdo et al., 1991) using $(\text { TTAGGG })_{5} /(\mathrm{CCCTAA})_{5}$ and $(\text { TTAGG })_{5} /(\mathrm{CCTAA})_{5}$ as primers, respectively. All probes were DIG-labelled by random priming (PCR products) or nick translation (ribosomal sequences) according to manufacturer's instructions (Roche, Mannheim, Germany). Slides were pre-treated with RNAse A $(200 \mu \mathrm{g} / \mathrm{ml})$ 
and the chromosomes denatured in $70 \%$ formamide $/ 2 \times \mathrm{SSC}$ for $4 \mathrm{~min}$ at $72^{\circ} \mathrm{C}$. Probes were denatured for $5 \mathrm{~min}$ at $80^{\circ} \mathrm{C}$. Each slide was hybridized with $25 \mu \mathrm{l}$ of the hybridization mixture containing $120 \mathrm{ng}$ of labelled and denatured probe dissolved in hybridization solution $(50 \%$ formamide, $10 \%$ dextran sulfate, $2 \times \mathrm{SSC}, 50 \mathrm{mM}$ sodium phosphate). Hybridization was allowed to proceed overnight in a moist plastic chamber at $37^{\circ} \mathrm{C}$. Slides were washed at $42^{\circ} \mathrm{C}$. Immunodetection of each probe was performed using an antibody cascade (anti-DIG, anti-mouse Ig-DIG and anti-DIG-FITC conjugated) from the Fluorescent Antibody Enhancer Set for DIG Detection (Roche, Cat. No. 1 768 506). Slides were mounted in antifade solution with propidium iodide (PI) $(3 \mu \mathrm{g} / \mathrm{ml})$ and viewed under a Leica filter set I3 (BP 450-490, LP 515), which allowed the simultaneous visualization of fluorescein-labelled hybrid (yellow) and chromosomal DNA (red).

Sequential treatment with Ag-NOR staining and rDNA FISH was carried out following Zurita et al. (1998), with slight modifications. An additional twenty males were used in a second stage of the analysis as a source of chromosome spreads from testicular lobes fixed in $3: 1$ solution of ethanol: acetic acid, and then stored at $-20^{\circ} \mathrm{C}$

\section{RESULTS}

The standard karyotype of $P$. ortolaniae males is $2 \mathrm{n}=$ 19 (9AA + X0) acrocentric chromosomes (Mansueto \& Vitturi, 1989).

Hybridizations with the ribosomal probes from $P$. lividus and D. melanogaster (employed in different experiments) gave better results with meiotic chromosomes (pachytene, diplotene and diakinesis stages). Both probes always gave the same results so that their hybridization signals were not distinguished from one another. At diakinesis, rDNA clusters were clearly evident on one ring bivalent ( 28 spreads evaluated). Hybridization signal was spread over a large portion of both homologous chromosomes but not uniformly stained due to the presence of numerous grains, which were more (Fig. 1A) or less (Figs $1 \mathrm{~B}, \mathrm{C})$ closely joined. When at the pachytene and diplotene stages a greatly enlarged rDNA-bearing bivalent appeared, it was possible to observe that the labelled area was organized in a multiclustered fashion consisting of three distinct serial regions designated as $r_{1}, r_{2}$ and $r_{3}$. The first band $\left(r_{1}\right)$ was adjacent to the centromere and appeared to be slightly larger than $r_{2}$ and $r_{3}$, which were nearly similar in size and located interstitially (see also Fig. 1D). The finding that the $P$. ortolaniae genome has one NOR (scattered in three regions along the chromosomal body) on one chromosome pair was supported by the observation that there is a maximum of two nucleoli in FISH-treated interphase nuclei (data not shown).

NOR activity was examined in primary (Fig. 3A) and secondary (data not shown) spermatocytes or spermatogonia (Fig. 3B) by means of silver impregnation. In every stage investigated (29 metaphase I and 21 mitotic metaphases), there were no deposits other than small silver aggregates in the centromeric regions of all chromosomes. This difference between the FISH and Ag-NOR patterns of staining was confirmed by sequentially treating with the two banding methods (compare Figs $3 \mathrm{~A}$ and $3 \mathrm{C}$ ). In the second stage of the cytogenetic analysis, the response of the ribosomal DNA regions to silver impregnation in the mitotic and meiotic plates from twenty additional males was investigated. In eighteen out of twenty specimens, the silver patterns consistently confirmed NORs do not stain with Ag. Only in five mitotic plates from two individuals, did the NOR-bearing chromosome pair show distinct silver dots indicating the presence of a centromere and $r_{1}$, respectively. In any case, $r_{2}$ and $r_{3}$ were consistently silver negative (Fig. 3D).

FISH with the vertebrate-type (TTAGGG) $)_{n}$ telomeric sequence failed to produce any hybridization signals (data not shown), whereas after hybridization with the pentamer (TTAGG) $)_{n}$ - which occurs in many arthropods, including insects (Okazaki et al., 1993; Sahara et al., 1999) - diakinetic bivalents showed typically labelled terminal areas (Fig. 2).

\section{DISCUSSION}

rDNA FISH consistently mapped major ribosomal sites (18S-28S rDNA) at three distinct regions $\left(r_{1}, r_{2}, r_{3}\right)$, organized in a multiclustered fashion on both homologues of one bivalent. As confirmed by the maximum number of nucleoli (two) per cell in interphase nuclei, the genome of $P$. ortolaniae is characterized by a single NOR chromosome pair. Unlike FISH, silver staining reveals centromeric Ag-aggregates on all chromosomes during major spermatogenetic stages, whereas there are hardly any silver deposits on the corresponding hybridized regions. Exceptionally, on some plates $(<3 \%$; five out of more than 200 plates analysed) silver staining revealed the $r_{1}$ cluster, in addition to the centromere; $r_{2}$ and $r_{3}$ were consistently Ag-negative. As already reported for other animals (Pendás et al., 1994; Sánchez et al., 1995; Vitturi et al., 2001; Colomba et al., 2006), as well as P. ortolaniae, fluorescence in situ hybridization is a uniquely reliable tool for the physical mapping of major ribosomal genes.

There is no technical bias in our findings mainly because conventional fixation (ethanol-acetic acid) and staining, on which silver stainability is strongly dependent (Hubbel, 1985), were used in our experiments. Moreover, in similar experiments, NORs were regularly revealed in the worms Eisenia foetida (Oligochaeta) (Vitturi et al., 2000a) and Ophryotrocha macrovifera (Polychaeta) (Vitturi et al., 2000b) chosen as positive controls (data not shown). We also exclude the possibility that FISH may have detected DNA other than that of ribosomal genes, because the two rDNA sequences (from such distant species as an insect and an echinoderm) used as separate probes resulted in similar hybridization patterns. Therefore, considering the reliability of our results, unusual features of the chromosome complement of $P$. ortolaniae, such as (i) the distribution of rDNA clusters along the chromosomes; (ii) an extremely weak silver staining of ribosomal sites at spermatogonial metaphases; and (iii) the overall silver stainability of centromeric regions during all major meiotic stages, deserve to be noted.

Based on the distribution of ribosomal DNA (scattered in three regions), the discrete length of the hybridized 
areas, interspersion and sequence fidelity of these moderately repeated sequences, it is likely that transposition may be responsible for the multiplication and dispersion of rDNA clusters.

Considering the poor response of rDNA regions to silver impregnation, it is very unlikely that all ribosomal clusters are transcriptionally inactive, but as already reported for other taxa (e.g. see Libertini et al., 2000; Vitturi et al., 2004), silver staining is ineffective, possibly due to the presence of a "protective shield", whose nature is still unknown (maybe proteinaceous or cytoplasmic), which prevents silver accessibility and precipitation. Our results suggest that from time to time (in a very low percentage of cases) cluster $r_{1}$ can be revealed by silver staining, whereas there is no clear explanation for the consistently negative reaction of $r_{2}$ and $r_{3}$. Three non-mutually exclusive hypotheses can be considered: (i) $r_{2}$ and $r_{3}$ remain unstained due to the presence of a molecular barrier. Moreover, taking into account the relative sizes of the three rDNA regions, any parameter preventing the detection of $r_{1}$ is likely to have a more powerful effect on shorter segments, such as $r_{2}$ and $r_{3}$; (ii) $r_{2}$ and $r_{3}$ are transcribed but remain undetectable since the level of silver staining is below the detection threshold; (iii) $r_{2}$ and $r_{3}$ clusters are transcriptionally inactive or at least not sufficiently decondensed (Clavaguera et al., 1983).

The intense silver staining of centromeres during spermatogenesis - an event quite unusual in vertebrates but commonly observed, although only at metaphase I, in invertebrates of very distant taxa (Vitturi et al., 2004 and references therein) - might be attributed to the occurrence of argentophilic acidic proteins different from those associated with ribosomal gene clusters. In fact, it is well known that silver nitrate impregnates proteinaceous chromosome structures including kinetochores, NORs, synaptonemal complexes, chromatid cores and mitotic chromosome scaffolds (Rufas et al., 1994 and references therein). One possibility is that, as already described for other grasshoppers, silver preferentially stains kinetochores, although, contrary to Rufas and co-workers' protocol, we did not impregnate overnight.

Our results confirm that, as expected, $P$. ortolaniae chromosomes share with other arthopods, including most insect groups, the (TTAGG) ${ }_{n}$ motif in the telomeric DNA. It is well known that (TTAGG) ${ }_{n}$ is a widespread, although not the only telomere motif in insects (Okazaki et al., 1993; Sahara et al., 1999; Frydrychová et al., 2004). In particular, it is widely reported that the (TTAGG) $)_{n}$ motif is conserved in the main lineages of insects with only occasional exceptions outside Endopterygota, which show a certain heterogeneity with respect to the occur-

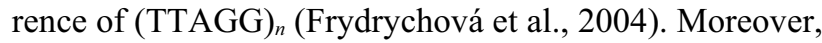
Vítková et al. (2005) report the presence of (TTAGG) ${ }_{n}$ in all main arthopod clades. This interesting finding strongly supports the hypothesis that the sequence is the ancestral telomere motif not only for insects, but for all arthopods. It is conceivable that (TTAGG) $)_{n}$ evolved from the so called "vertebrate" repeat (TTAGGG) ${ }_{n}$, which appears to be ancestral for all Metazoa (Traut et al., 2007).
Finally, it is interesting to compare the results of this study with those reported for grasshoppers either belonging to the family Pamphagidae (Cabrero et al., 1987; López-León et al., 1999) or even the same genus, Pamphagus (Camacho et al., 1981; Cabrero et al., 1985). Unlike $P$. ortolaniae, these species showed silver aggregates easily identifiable as active NORs at both mitotic and meiotic stages without, as far as we know, any additional centromeric Ag-deposits. All this suggests that $P$. ortolaniae might be an interesting biological model for studying rDNA organization and function. For this, it would be necessary to undertake a molecular analysis of the genome of $P$. ortolaniae and do a cytogenetic and molecular investigation of other Pamphagus species and genera of the family Pamphagidae.

ACKNOWLEDGEMENTS. We are grateful to S. Pisciotta and P. D'Agati for help with sample collection; to R. Barbieri (University of Palermo) for providing the $18 \mathrm{~S}$ rDNA probe from the sea urchin; to J. Méndez and A. Insua (Universidade da Coruña, Spain) for kindly providing glycerol stock of ribosomal DNA probe pDm238 from D. melanogaster, to M. Rampin (Università di Padova, Italy) for making and DIG labelling pDm238 ribosomal and (TTAGGG) $)_{\mathrm{n}}$ telomeric probes, to M.C. Maone for helping with experiments and, finally, to two anonymous referees whose suggestions have significantly improved the manuscript. This work was supported by a MURST grant (ex $60 \%$ ) to R. Vitturi and C. Mansueto.

\section{REFERENCES}

Cabrero J., Camacho J.P.M. \& Pascual F. 1985: Cytotaxonomic studies on Pamphagid genus Eumigus. Detection of two chromosomal races in E. monticola (Rambur) (Insecta, Orthoptera). Caryologia 38: 1-12.

Cabrero J., Alichè J.D. \& Camacho J.P.M. 1987: Effects of B chromosomes on the activity of nucleolar organizer regions in the grasshopper Eyprepocnemis plorans: activation of a latent nucleolar organizer region on a $\mathrm{B}$ chromosome fused to an autosome. Genome 29: 116-121.

Cabrero J., Bakkali M., Bugrou A., Warchalowska-Sliwa E., López León M.A., Perfectti F. \& CAmacho J.P.M. 2003: Multiregional origin of B chromosomes in the grasshopper Eyprepocnemis plorans. Chromosoma 112: 207-211.

Camacho J.P.M., Cabrero J. \& Viseras E. 1981: C-heterochromatin variation in the genus Eumigus (Orthoptera, Pamphagoidea). Genetica 56: 185-188.

Cantone M., Barbieri R., Duro G. \& Giudice G. 1993: Sequence analysis of the rDNA spacer of Paracentrotus lividus and observations about pre-RNA processing. NTS sequence of Paracentrotus lividus rDNA. Mol. Biol. Reports 18: $177-182$.

Clavaguera A., Querol E., Dolors C., Genesca J. \& Egozcue J. 1983: Cytochemical studies on the nature of NOR (nucleolus organizer regions) silver stainability. Cell. Mol. Biol. 29: 255-259.

Colomba M., Vitturi R., Volpe N., Lannino A. \& Zunino M. 2004: Karyotype, banding and rDNA FISH in the scarab beetle Anoplotrupes stercorosus (Coleoptera Scarabaeoidea: Geotrupidae). Description and comparative analysis. Micron 35: 717-720.

Colomba M., Vitturi R., Libertini A., Gregorini A. \& Zunino M. 2006: Heterochromatin of the scarab beetle Bubas bison (Coleoptera: Scarabaeidae) II. Evidence for AT-rich compart- 
mentalization and a high amount of rDNA copies. Micron 37: $47-51$.

Cusimano G. \& Massa B. 1977: Panfagidi nuovi delle isole circumsiciliane (Orthoptera Acridoidea). Atti Acad. Sci. Lett. Arti Palermo Serie IV, XXXV, Parte I: 1-22.

Frydrychová R., Grossmann P., Trubač P., VítKová M. \& MAREC F. 2004: Phylogenetic distribution of TTAGG telomeric repeats in insects. Genome 47: 163-178.

Howell W.H. \& Black D.A. 1980: Controlled silver-staining of nucleolus organizer regions with a protective colloidal developer: a 1-step method. Experientia 36: 1014.

HuBbell H.R. 1985: Silver staining as an indicator of active ribosomal genes. Stain Technol. 60: 285-294.

Ijdo J.W., Wells R.A., Baldini A. \& Reeders S.T. 1991: Improved telomere detection using a telomere repeat probe (TTAGGG) $)_{n}$ generated by PCR. Nucl. Acids Res. 19: 4780.

JoRDAN J. 1987: At the heart of the nucleolus. Nature 329 : 489-490.

Keller I., Chinauan-Marquier I.C., Veltsos P. \& Nichols R.A. 2006: Ribosomal DNA in the grasshopper Podisma pedestris: escape from concerted evolution. Genetics 164: 863-874.

Levan A., Fredga K. \& SAndberg A.A. 1964: Nomenclature for centromeric position of chromosomes. Hereditas 52: 201-220.

Libertini A., Colomba M.S. \& VitTuRi R. 2000: Cytogenetics of the amphipod Jassa marmorata (Corophioidea: Ischyroceridae): karyotype morphology, chromosome banding, fluorescent in situ hybridization, and nuclear DNA content. $J$. Crust. Biol. 20: 350-356.

López-León M.D., CABrero J. \& CAMAChO J.P.M. 1999: Unusual high amount of inactive ribosomal DNA in the grasshopper Stauroderus scalaris. Chromosome Res. 7: 83-88.

Lorite P., Aranega E., Luque F. \& Palomeque T. 1997: Analysis of the nucleolar organizing regions in the ant Tapinoma nigerrimum (Hymenoptera, Formicidae) Heredity 78: $578-582$.

Mansueto C. \& Vitturi R. 1989: NORs location and C-banding in spermatogenesis of Pamphagus ortolanii (Orthoptera, Pamphagidae). Caryologia 42: 303-311.

Miller D.A., Dev V.G., Tantravahi R. \& Miller O.J. 1976: Suppression of human nucleolus organizer activity in mousehuman somatic hybrid cells. Exp. Cell Res. 101: 235-243.

NATH J.\& Johnson K.L. 2000: A review of fluorescence in situ hybridization (FISH): current status and future prospects. Biotech. Histochem. 75: 54-78.

Nomoto Y., Hirai M. \& Ueshima R. 2001: Cloning of molluscan telomere DNA with (TTAGGG) $)_{\mathrm{n}}$ repeat and its chromosomal location in the freshwater snail Biwamelania habei. Zool. Sci. 18: $417-422$.

Okazaki S., Tsuchida K., Maekawa M., Ishikawa M. \& FujIWARA H. 1993: Identification of a pentanucleotide telomeric sequence (TTAGG) $)_{n}$ in the silkworm Bombyx mori and in other insects. Mol. Cell Biol. 13: 1424-1432.

Pendás A.M., Morán P., Freije J.P. \& Garcia-Vazquez E. 1994: Chromosomal mapping and nucleotide sequence of two tandem repeats of Atlantic salmon. Cytogenet. Cell Genet. 67: $31-36$.

Roiha H., Miller J.R., Woods L.C. \& Glover D.M. 1981: Arrangements and rearrangements of sequences flanking the two types of rDNA insertion in D. melanogaster. Nature 290: 749-753.
Rufas J.S., Mazzella C., García de la Vega C. \& Suja J.A. 1994: Ultrastructural detection of kinetochores by silver impregnation. Chromosome Res. 2: 369-375.

Sahara K., Marec F. \& Traut W. 1999: TTAGG telomeric repeats in chromosomes of some insects and other arthropods. Chromosome Res. 7: 449-460.

Sánchez A., Jimenez R., Burgos M., Stitou S., Zurita F. \& Diaz DE LA GuARdia R. 1995: Cytogenetic peculiarities in the Algerian hedgehog: silver stains not only NORs but also heterochromatic blocks. Heredity 75: 10-16.

SLIJEPCEVIC P. 1998: Telomeres and mechanisms of Robertsonian fusion. Chromosoma 107: 136-140.

Souza M.J. \& Melo N.F. 2007: Chromosome study in Schistocerca (Orthoptera - Acrididae - Cyrtacanthacridinae): karyotypes and distribution patterns of constitutive heterochromatin and nucleolus organizer regions (NORs). Genet. Mol. Biol. 30: $54-59$.

Souza M.J., Rufas J.S. \& Orellana J. 1998: Constitutive heterochromatin, NOR location and FISH in the grasshopper Xyleus angulatus (Romaleidae). Caryologia 51: 73-80.

Swiger R.R. \& Tucker J.D. 1996: Fluorescent in situ hybridization: a brief review. Environ. Mol. Mutagen. 27: 245-254.

Traut W., Szczepanowski M., VitKová M., Opitz C., Marec F. \& ZRZAVÝ J. 2007: The telomere repeat motif of basal Metazoa. Chromosome Res. 15: 371-382.

Vítková M., Král J., Traut W., Zrzavý J. \& Marec F. 2005: The evolutionary origin of insect telomeric repeats, (TTAGG) ${ }_{n}$. Chromosome Res. 13: 145-156.

VITTURI R. 1992: Conventionally stained chromosomes, constitutive heterochromatin and nucleolus organizer regions in Milax nigricans (Gastropoda, Pulmonata). Chromatin 1: 147-155.

Vitturi R., Mansueto C. \& Di Paola Ficarella F. 1993: Heterochromatin variation in four species of the genus Pamphagus (Orthoptera, Pamphagidae) analyzed by C-banding. Biol. Zentbl. 112: 335-341.

Vitturi R., Colomba M.S., Pirrone A.M. \& Libertini A. 2000a: Physical mapping of rDNA genes, (TTAGGG)n telomeric sequence and other karyological features in two earthworms of the family Lumbricidae (Annelida: Oligochaeta). Heredity 85: 203-207.

Vitturi R., Ramella L., Colomba M.S., Caputo V. \& Sella G. 2000b: NOR regions of polychaete worms of the genus Ophryotrocha studied by chromosome banding techniques and FISH. J. Hered. 91: 18-23.

Vitturi R., Colomba M.S., Mandrioli M., Pirrone A.M., GRISPO G. \& BARBIERI R. 2001: 18S-28S rDNA is interspersed throughout heterochromatin in the millipede Enologus oxypygum (Diplopoda). Genetica 110: 151-155.

Vitturi R., Sineo L., Volpe N., Lannino A. \& Colomba M. 2004: Repetitive DNAs: association of ribosomal (18S-28S and $5 \mathrm{~S}$ rDNA) and (TTAGGG) $)_{\mathrm{n}}$ telomeric sequences in the slug Milax nigricans (Mollusca: Gastropoda: Pulmonata). Micron 35: 255-260.

Zurita F., Jiménez R., Burgos M. \& Diaz de la Guardia R. 1998: Sequential silver staining and in situ hybridization reveal a direct association between rDNA levels and the expression of homologous nucleolar organizing regions: a hypothesis for NOR structure and function. J. Cell Sci. 111: 1433-1439.

Received March 22, 2007; revised and accepted August 1, 2007 